TITLE:

ON CHAETOGNATHS AND APPENDICULARIANS COLLECTED IN THE CENTRAL PART OF THE INDIAN OCEAN

$\operatorname{AUTHOR(S):~}$

Tokioka, Takasi

CITATION:

Tokioka, Takasi. ON CHAETOGNATHS AND APPENDICULARIANS COLLECTED IN THE CENTRAL PART OF THE INDIAN OCEAN. PUBLICATIONS OF THE SETO MARINE BIOLOGICAL LABORATORY 1956, 5(2): 197-200

ISSUE DATE:

1956-06-30

URL:

http://hdl.handle.net/2433/174558

RIGHT: 


\title{
ON CHAETOGNATHS AND APPENDICULARIANS COLLECTED IN THE CENTRAL PART OF THE INDIAN OCEAN
}

\author{
TAKASI TOKIOKA
}

Seto Marine Biological Laboratory, Sirahama

With a Chart and 2 Tables

Chaetognaths and appendicularians were selected out of 26 plankton samples collected $^{2)}$ by the Syunkotu-maru, a training ship of the Simonoseki College of Fisheries, at 19 stations in the central part of the Indian Ocean, south-west to the Maldive Islands, during the period from Dec. 11, 1954 to Jan. 16, 1955 and offered to me for the identification by courtesy of Mr. S. Tsuruta of the Simonoseki College of Fisheries, to whom I express here my hearty thanks for his kindness. The situation of respective stations and the date of each sampling are indicated at the end of this article.

\section{Chaetognaths}

The material comprises the following 13 species. The detail of the occurrence is shown in Table 1.

Species

1. Sagitta hexaptera

2. Sagitta lyra

3. Sagitta enflata

4. Sagitta robusta

5. Sagitta ferox

6. Sagitta bedoti

7. Sagitta serratodentata pacifica

8. Sagitta regularis

9. Sagitta bedfordii

10. Sagitta minima

11. Pterosagitta draco
Number of individuals

58

15

579

30

19

381

766

267

39

216

133
Percentage

2

1

22

1

1

14

29

10

1

8

5

1) Contributions from the Seto Marine Biological Laboratory, No. 276.

2) KrTAHARA's quantitative plankton net was hauled vertically at the speed of $50 \mathrm{~cm}$ per second. This is a modified HENSEN's net reduced in a smaller size, $25 \mathrm{~cm}$ in diameter of the mouth and $100 \mathrm{~cm}$ in length, and stretched with sieve silk, GG 68 .

Publ. Seto Mar. Biol. Lab., V (2), 1956. (Article 10) 
12. Krohnitta subtilis

13. Krohnitta pacifica

14. Damaged individuals or juv.

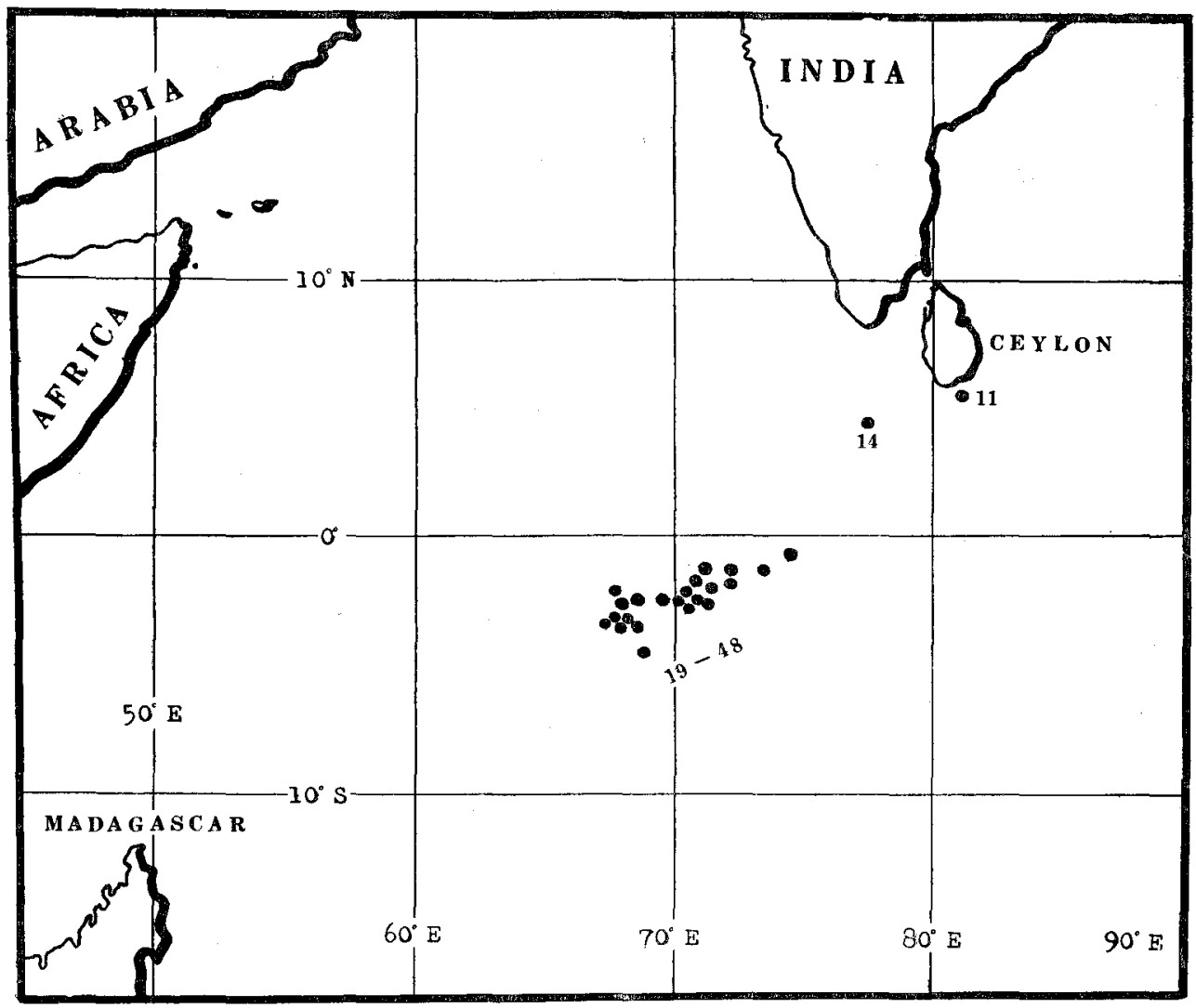

Chart 1. Map showing stations of sampling.

Sag. serratodentata pacifica and Sag. enflata are the dominant species and followed by Sag. bedoti and Sag. regularis. Pterosag. draco occurs frequently, but rather in a small number. Sag. minima occurs less frequently than Pterosag. draco, but its population is denser than that of the latter, when it is met with.

The surveyed area is located near, very slightly north of, the Area I of the "Sea lark" Expedition" in 1905. But, the difference found between the chaetognath faunas clarified by that expedition and the present survey seems to be rather remark-

1) Eurfield, S. T. (1926): The Percy Sladen Trust Expedition to the Indian Ocean in 1905. No. V. The Chaetognatha of the "Sea lark" Expedition. Trans. Linn. Soc. London, Zool. Vol, XIX, Part 1. 
able: Sag. bedoti occurred much oftener in the present material than in the "Sea lark" material and Sag. minima was found in a considerable quantity in the former, although it was quite absent in the latter. Comparing the present results with those of the expedition in May-June 1954 in the waters around the Marshall Islands ${ }^{13}$ in the Pacific, the relative abundance of Sag. serratodentata pacifica, Sag. bedoti, Sag. regularis and Sag. minima and the scarcity of Sag. bipunctata in the central part of the Indian Ocean seem to be very impressive.

\section{Appendicularians}

The following 23 species were found in the material.

Species

1. Oikopleura longicauda

2. Oikopleura fusiformis

3. Oikopleura fusiformis f. cornutogastra

4. Oikopleura intermedia

5. Oikopleura gracilis

6. Oikopleura graciloides

7. Oikopleura dioica

8. Oikopleura rufescens

9. Oikopleura parva

10. Oikopleura cophocerca

11. Oikopleura albicans

12. Oikopleura spp. (damaged)

13. Megalocercus huxleyi

14. Stegosoma magnum

15. Pelagopleura verticalis

16. Fritillaria haplostoma

17. Fritillaria formica f. digitata

18. Fritillaria fraudax

19. Fritillaria gracilis

20. Fritillaria pellucida

21. Fritillaria borealis f. sargassi (large individual)

$21 a$. (small individual)

22. Fritillaria borealis

f. intermedia
Number of Individuals 371

91

12

1

7

4

1

6

66

11.

34

16

65

3

42

5

2

11

1

1

2

6

1

4

1

28

4

1

1) TokiokA, T. (1955): On some plankton animals collected by the Syunkotu-maru in MayJune 1954. I Chaetognatha. Publ. Seto Mar. Biol. Lab., IV (2-3). 
23. Fritillaria megachile

24. Fritillaria spp. (damaged)

25. Appendicularia sicula

\begin{tabular}{rr}
2 & - \\
4 & 1 \\
4 & 1 \\
\hline 788 &
\end{tabular}

Oik. longicauda is the dominant-most species of all and followed by Oik. fusiformis. Oik. rufescens occurs also frequently, while Oik. cophocerca and Steg. magnum are found less frequently and only in a small amount. The remarkable increase of the percentage of Oik. fusiformis and the decrease of that of Oik. fusiformis f. cornutogastra in the Indian Ocean are noticeable, when the present results are compared with those of the survey in the Arafura Sea. Both Oik. dioica and App. sicula are decreased considerably in the central part of the Indian Ocean. The detail of the occurrence of respective species may be understood in Table 2.

\begin{tabular}{|c|c|c|c|c|}
\hline $\begin{array}{l}\text { Station } \\
\text { Number }\end{array}$ & Latitude & Longitude & & ate \\
\hline 11 & $5^{\circ} 37^{\prime} \mathrm{N}$ & $81^{\circ} 07^{\prime} \mathrm{E}$ & 1954 & Dec. 11 \\
\hline 19 & $0^{\circ} 43^{\prime} \mathrm{S}$ & $74^{\circ} 36.5^{\prime} \mathrm{E}$ & & 15 \\
\hline 22 & $1^{\circ} 07.8^{\prime} \mathrm{S}$ & $71^{\circ} 15^{\prime} \mathrm{E}$ & & 18 \\
\hline 23 & $1^{\circ} 46^{\prime} \mathrm{S}$ & $71^{\circ} 01^{\prime} \mathrm{E}$ & & 19 \\
\hline 24 & $2^{\circ} 41^{\prime} \mathrm{S}$ & $70^{\circ} 35^{\prime} \mathrm{E}$ & & 20 \\
\hline 25 & $2^{\circ} 15^{\prime} \mathrm{S}$ & $70^{\circ} 31^{\prime} \mathrm{E}$ & & 21 \\
\hline 33 & $2^{\circ} 11.5^{\prime} \mathrm{S}$ & $67^{\circ} 55.2^{\prime} \mathrm{E}$ & 1955 & Jan. 6 \\
\hline 34 & $2^{\circ} 26^{\prime} \mathrm{S}$ & $68^{\circ} 33^{\prime} \mathrm{E}$ & & 7 \\
\hline 35 & $3^{\circ} 02^{\prime} \mathrm{S}$ & $68^{\circ} 10^{\prime} \mathrm{E}$ & & 8 \\
\hline 36 & $2^{\circ} 28^{\prime} \mathrm{S}$ & $68^{\circ} 01^{\prime} \mathrm{E}$ & & 9 \\
\hline 37 & $3^{\circ} 19^{\prime} \mathrm{S}$ & $68^{\circ} 19^{\prime} \mathrm{E}$ & & 10 \\
\hline 38 & $4^{\circ} 35^{\prime} \mathrm{S}$ & $68^{\circ} 58^{\prime} \mathrm{E}$ & & 11 \\
\hline 39 & $3^{\circ} 17^{\prime} \mathrm{S}$ & $68^{\circ} 00^{\prime} \mathrm{E}$ & & 12 \\
\hline 40 & $3^{\circ} 10^{\prime} \mathrm{S}$ & $67^{\circ} 59^{\prime} \mathrm{E}$ & & 13 \\
\hline 41 & $3^{\circ} 17^{\prime} \mathrm{S}$ & $67^{\circ} 43^{\prime} \mathrm{E}$ & & 14 \\
\hline 43 & - & - & & 15 \\
\hline 44 & - & - & & 15 \\
\hline 45 & $2^{\circ} 31^{\prime} \mathrm{S}$ & $70^{\circ} 18.5^{\prime} \mathrm{E}$ & & 15 \\
\hline 47 & $2^{\circ} 02^{\prime} \mathrm{S}$ & $71^{\circ} 33^{\prime} \mathrm{E}$ & & 16 \\
\hline
\end{tabular}


Table 1. Detail of the occurrence of chaetognaths.

F. O.......Frequency of occurrence, M.N./H......Mean number of individuals per haul.

\begin{tabular}{|c|c|c|c|c|c|c|c|c|c|c|c|c|c|c|c|c|c|c|c|c|c|c|c|c|c|c|c|c|}
\hline \multirow{2}{*}{$\begin{array}{c}\text { Station Number } \\
\text { Haul } \\
\text { Distance }\end{array}$} & \multicolumn{2}{|c|}{11} & \multirow{2}{*}{$\frac{19}{8}$} & \multicolumn{2}{|c|}{22} & \multirow{2}{*}{$\frac{23}{8}$} & \multicolumn{2}{|c|}{24} & \multirow{2}{*}{$\begin{array}{l}25 \\
8 \\
0 \\
0\end{array}$} & \multicolumn{2}{|c|}{33} & \multicolumn{2}{|c|}{34} & \multirow{2}{*}{\begin{tabular}{|c|}
35 \\
8 \\
8 \\
0
\end{tabular}} & \multirow{2}{*}{$\begin{array}{l}36 \\
8 \\
8 \\
0 \\
0\end{array}$} & \multicolumn{2}{|c|}{37} & \multirow{2}{*}{$\frac{38}{\substack{8 \\
0 \\
0}}$} & \multirow{2}{*}{$\begin{array}{l}39 \\
8 \\
\\
0 \\
0\end{array}$} & \multicolumn{2}{|c|}{40} & \multirow{2}{*}{$\begin{array}{l}41 \\
8 \\
0 \\
0\end{array}$} & \multirow{2}{*}{$\frac{43}{\substack{0 \\
1 \\
0}}$} & \multirow{2}{*}{\begin{tabular}{l|}
44 \\
8 \\
0 \\
1 \\
0
\end{tabular}} & \multirow{2}{*}{\begin{tabular}{|l|}
45 \\
8 \\
\\
0 \\
\end{tabular}} & \multirow{2}{*}{$\frac{47}{\substack{8 \\
\text { 1 } \\
0}}$} & \multirow[b]{2}{*}{ F. O. } & \multirow{2}{*}{$\frac{\text { M.N. }}{\text { H. }}$} \\
\hline & $\mid \begin{array}{l}8 \\
1 \\
1 \\
0\end{array}$ & $\cdots$ & & $\begin{array}{l}8 \\
\stackrel{1}{1} \\
0\end{array}$ & \begin{tabular}{|l|}
8 \\
10 \\
1 \\
0
\end{tabular} & & 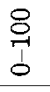 & n. & & $\begin{array}{l}8 \\
7 \\
0 \\
0\end{array}$ & $\begin{array}{l}8 \\
0 \\
0\end{array}$ & $\begin{array}{l}8 \\
0 \\
1 \\
0\end{array}$ & $\begin{array}{l}8 \\
\\
0\end{array}$ & & & $\begin{array}{l}8 \\
8 \\
1 \\
0\end{array}$ & $\begin{array}{l}\mathbb{8} \\
1 \\
0\end{array}$ & & & $\dot{\vec{n}}$ & $\begin{array}{l}8 \\
\\
0\end{array}$ & & & & & & & \\
\hline Sag. hexaptera & & & & & 6 & 2 & & & 1 & 4 & & 2 & 2 & & & & 4 & 3 & 1 & 6 & 2 & 3 & 5 & 1 & 9 & 7 & $16 / 26$ & 3.6 \\
\hline Sag. lyra & & & & & & & 1 & & & & & 4 & & & & & 3 & 1 & & & 1 & & 3 & 2 & & & 7 & 2.1 \\
\hline Sag. enflata & 1 & 1 & 4 & 15 & 35 & 25 & 21 & 5 & 45 & 43 & 46 & 51 & 3 & 24 & 10 & 8 & 17 & 22 & 33 & 7 & 23 & 32 & 12 & 64 & 18 & 14 & 26 & 22.3 \\
\hline Sag, robusta & & & & 2 & 4 & 1 & 2 & & & 5 & & 2 & & 1 & 6 & 1 & & 1 & & 2 & & & 1 & 1 & & 1 & 14 & 2.1 \\
\hline Sag. ferox & 1 & & 1 & & 2 & & & & & & 1 & & 1 & 2 & & & & & 4 & 1 & 1 & & 3 & 2 & & & 11 & 1.7 \\
\hline Sag. bedoti & & 1 & 4 & 6 & 15 & 27 & 10 & & 18 & 43 & 14 & 26 & 9 & 9 & & & 4 & 6 & 8 & & 9 & 4 & 31 & 74 & 45 & 18 & 21 & 18.1 \\
\hline $\begin{array}{c}\text { Sag. serratodentata } \\
\text { pacifica }\end{array}$ & 10 & 3 & 12 & 13 & 91 & 41 & 10 & 5 & 27 & 36 & 73 & 90 & 9 & 54 & 8 & 7 & 43 & 13 & 17 & 5 & 18 & 32 & 32 & 45 & 40 & 32 & 26 & 29.5 \\
\hline Sag. regularis & 5 & 3 & 1 & & 5 & 6 & 7 & 1 & 10 & 16 & 2 & 6 & 2 & 2 & 66 & 1 & 5 & 13 & 10 & 12 & 12 & 13 & 19 & 22 & 25 & 3 & 25 & 10.7 \\
\hline Sag. bedfordii & & & & 2 & & & 1 & & 5 & & 2 & & & . & 1 & & & 14 & & & 1 & 5 & & 1 & 5 & 2 & 11 & 3.5 \\
\hline Sag. minima & & & & & & & 1 & & & 49 & 24 & 27 & 6 & 18 & & 5 & 9 & 8 & 15 & & 14 & 2 & 25 & 12 & 1 & & 15 & 14.4 \\
\hline Sag. spp. & & & & 2 & 4 & 1 & 4 & & & 5 & 1 & 2 & 2 & 1 & & 14 & & 1 & 2 & & & 1 & & 3 & & 1 & 15 & 2.9 \\
\hline Pserosag. draco & 5 & & 2 & 6 & 23 & 3 & 4 & & 5 & 10 & 4 & 10 & & 5 & & & 5 & 9 & 2 & & 1 & 2 & 12 & 9 & 6 & 10 & 20 & 6.7 \\
\hline Ktta. subtilis & & & & & 3 & 1 & 1 & & 1 & 2 & & 3 & 1 & 3 & & 1 & 7 & 3 & 1 & & 3 & 3 & & 5 & & 2 & 16 & 2.5 \\
\hline Ktta. pacifica & & & & 2 & & 1 & 3 & 1 & 4 & 9 & 5 & 8 & & & & 1 & 1 & 1 & & 1 & 1 & 2 & & 7 & 1 & & 16 & 3.0 \\
\hline Total & 22 & 8 & 24 & 48 & 188 & 108 & 65 & 12 & 116 & 222 & 172 & 231 & 35 & 119 & 91 & 38 & 98 & 95 & 93 & 34 & 86 & 99 & 143 & 248 & 150 & 90 & & \\
\hline
\end{tabular}


Table 2. Detail of the occurrence of appendicularians.

F. O.......Frequency of occurrence, M.N./H. ..... Mean number of individuals per haul.

\begin{tabular}{|c|c|c|c|c|c|c|c|c|c|c|c|c|c|c|c|c|c|c|c|c|c|c|c|c|c|c|c|c|}
\hline Station Number & 11 & & 19 & 22 & & 23 & 24 & & 25 & 33 & & 3 & & 35 & 36 & 37 & & 38 & 39 & 40 & & 41 & 43 & 44 & 45 & 47 & & \\
\hline $\begin{array}{c}\text { Haul } \\
\text { Distance }\end{array}$ & $\left|\begin{array}{ll}8 & 1 \\
7 & 1 \\
0 & 1\end{array}\right|$ & n. & $\begin{array}{l}8 \\
-1 \\
0\end{array}$ & $\begin{array}{l}8 \\
8 \\
1 \\
0\end{array}$ & $\begin{array}{c}8 \\
80 \\
0\end{array}$ & $\begin{array}{l}8 \\
\stackrel{1}{1} \\
0\end{array}$ & $\underset{1}{8}$ & ๙. & $\begin{array}{l}8 \\
\frac{8}{1} \\
d\end{array}$ & $\begin{array}{l}8 \\
\\
0\end{array}$ & $\begin{array}{l}8 \\
\\
1 \\
0\end{array}$ & $\begin{array}{l}8 \\
1 \\
0\end{array}$ & $\begin{array}{c}8 \\
\\
0\end{array}$ & $\begin{array}{l}8 \\
\stackrel{9}{9} \\
0\end{array}$ & $\begin{array}{l}8 \\
8 \\
0 \\
0\end{array}$ & $\begin{array}{l}8 \\
8 \\
\\
0\end{array}$ & $\begin{array}{l}\underset{\text { I }}{\text { N }} \\
0\end{array}$ & $\underset{1}{8}$ & $\begin{array}{l}8 \\
8 \\
0\end{array}$ & $\dot{\vec{G}}$ & $\begin{array}{l}8 \\
\stackrel{1}{1} \\
0\end{array}$ & $\begin{array}{l}8 \\
\stackrel{N}{N} \\
0\end{array}$ & $\begin{array}{l}8 \\
\stackrel{1}{1} \\
0\end{array}$ & $\begin{array}{l}8 \\
\stackrel{1}{1} \\
0\end{array}$ & $\begin{array}{l}8 \\
\frac{8}{6} \\
0\end{array}$ & $\begin{array}{l}8 \\
\stackrel{8}{0} \\
0 \\
0\end{array}$ & F.O. & $\frac{\mathrm{H} .}{\mathrm{H} .}$ \\
\hline Oik. longicauda & 1 & 1 & 36 & 8 & 7 & 12 & 3 & 15 & 3 & 3 & 63 & 8 & 2 & 17 & 3 & 2 & 19 & 9 & 15 & 43 & 11 & 32 & 10 & 18 & 20 & 10 & $26 / 26$ & 14.3 \\
\hline Oik. fusiformis & & & 5 & 3 & & 7 & 2 & 10 & 2 & 2 & 5 & 3 & & 3 & 1 & 1 & $\overline{4}$ & 3 & 3 & & 7 & 11 & 1 & 8 & 9 & 1 & 21 & 4.3 \\
\hline $\begin{array}{l}\text { Oik. fusiformis } \\
\text { f. cornutogastra }\end{array}$ & & & & & & & & & & & & & & & & & & & & & & 1 & & & & & 1 & 1 \\
\hline Oik. intermedia & & & & & & & & & & & & & & & & 2 & 5 & & & & & & & & & & 2 & 3.5 \\
\hline Oik. gracilis & & & & & & & & & & & & 2 & & & & & 2 & & & & & & & & & & 2 & 2 \\
\hline Oik. graciloides & & & & & & & & & & & & & & & & & & & & & & & & 1 & & & 1 & 1 \\
\hline Oik. dioica & & & & & & & & & & & & & & & & 1 & 1 & 3 & & 1 & & & & & & & 4 & 1.5 \\
\hline Oik. rufescens & & & 5 & & 3 & 3 & 2 & 13 & 1 & 3 & 14 & & 1 & 2 & & $\overline{2}$ & 3 & 1 & 1 & 3 & & 3 & 1 & 1 & 4 & & 19 & 3.5 \\
\hline Oik. parva & & & & & & & & 1 & & & & & & 1 & & & & & & & 3 & 1 & & & 2 & 3 & 6 & 1.8 \\
\hline Oik. cophocerca & & 1 & 1 & & & & 2 & 1 & & 3 & 4 & & & 3 & & 1 & 3 & & 1 & & 4 & 3 & & 1 & 4 & 2 & 15 & 2.3 \\
\hline Oik. albicans & & & & & 2 & & & & & & & & & 4 & & 1 & 5 & & & & & 2 & & & 1 & 1 & 7 & 2.3 \\
\hline Oik. spp. & 1 & & 1 & 8 & 6 & & 2 & $\overline{4}$ & 2 & 4 & 2 & 1 & & 5 & & 5 & 1 & & 5 & 2 & & 4 & & 5 & 4 & 3 & 19 & 3.4 \\
\hline Megal. huxleyi & & & & & & & & 1 & 1 & & & & & & & & 1 & & & & & & & & & & 3 & 1 \\
\hline Steg. magnum & & & & 1 & 5 & 1 & 5 & 5 & 5 & & & 1 & & 2 & & 6 & 1 & & 2 & 2 & 1 & 1 & & 2 & & 2 & 16 & 2.6 \\
\hline Pelag. verticalis & & & & & & & & & & & & & & 1 & & 2 & 1 & & & & & 1 & & & & & 4 & 1.3 \\
\hline Frit. haplostoma & & 1 & & & & & & 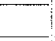 & & & & & & & & & & & & & & & & 1 & & & 2 & 1 \\
\hline $\begin{array}{l}\text { Frit. formica } \\
\quad \text { f. digitata }\end{array}$ & & & & & & & & 1 & & & & 2 & & 2 & & & 1 & & & & 1 & 2 & & & 2 & & 7 & 1.6 \\
\hline Frit. fraudax & & & & & 1 & & & & & & & & & & & & & & & & & & & & & & 1 & 1 \\
\hline Frit. gracilis & & & & & & & & & & & & & & & & & & & & & & & & & & 2 & 1 & 2 \\
\hline Frtt. pellucida & & & & & & & & & & 1 & $\overline{1}$ & & & & 1 & & 2 & & & & & 1 & & & & & 5 & 1.2 \\
\hline $\begin{array}{l}\text { Frit. borealis } \mathrm{f} . \\
\text { sargassi large ind. }\end{array}$ & & & & & & & & & 1 & & & & & & & & & & & & 1 & & & & & 2 & 3 & 1.3 \\
\hline $\begin{array}{l}\text { Frit. borealis } \mathrm{f} . \\
\text { sargassi small ind. }\end{array}$ & & 1 & & 4 & 14 & 2 & 1 & & & & & & & & & & & & & 1 & & 1 & & 1 & & 3 & 9 & 3.1 \\
\hline $\begin{array}{l}\text { Frit. borealis } \\
\quad \text { f. intermedia }\end{array}$ & & & & & & & & & & & & & & & & & & & & & & & & 1 & & & 1 & 1 \\
\hline Fruit. megachile & & & & & 1 & & & & & & & & & 1 & & & & & & & & & & & & & 2 & 1 \\
\hline Frit. spp. & & & & & 2 & & & & & & & & & & 1 & 1 & & & & & & & & & & & 3 & 1.3 \\
\hline App. sicula & & & & & & & & & & & & & & & & 1. & & & 1 & & 1 & 1 & & & & & 4 & 1 \\
\hline Total & 2 & 4 & 48 & 24 & 41 & 25 & 17 & 51 & 15 & 16 & 89 & 17 & 3 & 41 & 6 & 25 & 49 & 16 & 28 & 52 & 29 & 64 & 12 & 39 & 46 & 29 & & \\
\hline
\end{tabular}

